\title{
EFEITO DA DESRAMA ARTIFICIAL NA QUALIDADE DA MADEIRA DE CLONES DE EUCALIPTO EM SISTEMA AGROSSILVIPASTORIL ${ }^{1}$
}

\author{
Rodrigo Silva do Vale ${ }^{2}$, Renato Luiz Grisi Macedo ${ }^{3}$, Nelson Venturin ${ }^{3}$, Fábio Akira Mori e \\ Augusto Ramalho de Morais ${ }^{4}$
}

\begin{abstract}
RESUMO - O objetivo do presente trabalho foi avaliar o efeito da desrama artificial na qualidade da madeira de clones de eucalipto, em sistema agrossilvipastoril, na fazenda Riacho, pertencente à Companhia Mineira de Metais, município de Paracatu-MG, Brasil. O experimento foi instalado em dezembro de 1994, com o plantio das mudas de três clones de híbridos naturais de Eucalyptus camaldulensis Dehnh. (código 06) e de Eucalyptus urophylla S.T. Blake (código 13 e 44), no espaçamento de 10 x $4 \mathrm{~m}$. O delineamento experimental utilizado foi o de blocos casualizados, com três repetições. Os tratamentos foram dispostos em esquema de parcelas subdivididas, sendo os tratamentos primários constituídos pelos clones e os tratamentos secundários definidos por: $\mathrm{T}_{1}-$ sem desrama; $\mathrm{T}_{2}$ desrama a $2 \mathrm{~m}$; $\mathrm{T}_{3}$ - desrama a $4 \mathrm{~m}$; e $\mathrm{T}_{4}$ - desrama a $6 \mathrm{~m}$. Para avaliação da qualidade da madeira foram obtidos, no ano de 2000, a densidade básica da madeira, o número e o diâmetro dos nós, a nodosidade, o número de bolsas de resina e a conicidade das árvores. Os resultados obtidos mostraram que a densidade básica da madeira por árvore apresentou variações significativas entre os clones e as alturas de desrama. A densidade básica ao longo do tronco apresentou comportamento diferenciado perante os clones e as alturas de desrama. O número de nós e a nodosidade foram afetados pelas alturas de desrama. A conicidade e o número de bolsas de resina não foram afetados pelas alturas de desrama, apresentando diferença significativa somente entre os clones.
\end{abstract}

Palavras-chave: Desrama artificial, qualidade da madeira de clones de eucalipto e sistema agrossilvipastoril.

\section{EFFECT OF ARTIFICIAL PRUNING ON WOOD QUALITY OF EUCALYPT CLONES IN AN AGROSYLVOPASTORAL SYSTEM}

\begin{abstract}
This work aimed to evaluate the effect of artificial pruning on wood quality of eucalypt clones, under an agrosylvopastoral system, owned by the Riacho of the Companhia Mineira de Metais Farm in ParacatuMG, Brazil. The experiment was installed in December 1994 with the planting of seedlings of 3 clones of natural hybrids of Eucalyptus camaldulensis, Dehnh. (code 06) and Eucalyptus urophylla S.T.Blake (code 13 and 44), using a $10 \mathrm{~m} \times 4 \mathrm{~m}$ spacing. The experimental design used was randomized blocks with 3 repetitions. The treatments were established in split plots, with the primary treatments constituted by the clones and the secondary treatments defined as: $T_{1}$ - without pruning; $T_{2}$ - pruning up to $2 \mathrm{~m}$; $T_{3}$ - pruning up to $4 \mathrm{~m}$; $T_{4}$ - pruning up to $6 \mathrm{~m}$. For the evaluation of wood quality in the year of 2000, basic wood density, number and diameter of the knots, knottiness, number of pitch pockets and conicity of the trees were obtained. Basic wood density by tree presented significant variations among the clones and pruning heights. Basic density along the trunk presented a differentiated behavior among the clones and pruning heights. Number of knots and knottiness were affected by the pruning heights. Conicity and number of pitch pockets were not affected by the pruning heights, presenting a significant difference only among the clones.
\end{abstract}

Key words: $\quad$ Artificial pruning, wood quality of eucalypt clones, agrosylvopastoral system.

1 Recebido para publicação em 17.8.2001.

Aceito para publicação em 20.6.2002.

Parte integrante da Dissertação de Mestrado (DCF/UFLA) do primeiro autor. Pesquisa financiada pela FAPEMIG/FIEMG-IEL.

2 Eng.-Agrônomo M.S.; Doutorando em Ciência Florestal na Universidade Federal de Viçosa - UFV, <ds41781@ correio.ufv.br>;

${ }^{3}$ Professores do Departamento de Ciências Florestais (DCF) da Universidade Federal de Lavras - UFLA, Caixa Postal 37, 37200-

000 Lavras-MG; ${ }^{4}$ Professor do Departamento de Ciências Exatas da UFLA. 


\section{INTRODUÇÃO}

A utilização crescente das madeiras provenientes de reflorestamentos para serraria é evidente nos últimos anos, especialmente as dos gêneros Pinus e Eucalyptus.

A produção de madeira serrada de espécies de reflorestamento baseia-se significativamente no gênero Pinus. As espécies deste gênero não apresentam dificuldades no seu processamento. Entretanto, nos últimos anos, o estoque dessas florestas vem diminuindo consideravelmente, o que tem despertado o interesse de empresas florestais por espécies alternativas que apresentem rápido crescimento, sendo uma das opções o gênero Eucalyptus (Del Menezzi et al.,1998).

Atualmente, a busca do uso múltiplo das florestas e de melhor remuneração para a madeira tem incentivado o estabelecimento de estratégias por parte das empresas para a oferta de madeira serrada de eucalipto.

O gênero Eucalyptus é caracterizado pela sua grande capacidade de adaptação a diferentes condições ambientais, destacando-se também o seu rápido crescimento, devido às práticas silviculturais, ao manejo e, principalmente, ao melhoramento genético das espécies.

Em espaçamentos maiores verifica-se a plena disponibilidade de luz, conseqüentemente não se verifica o fenômeno de desrama natural. Logo, a prática silvicultural de desrama artificial torna-se essencial e necessária para atingir os objetivos de produção de madeira serrada de qualidade e preços superiores.

Um dos problemas de trabalhar com madeira reflorestada de eucalipto é a ocorrência de defeitos, como os nós e as bolsas de resina, também chamadas de veios de Kino. A resina é uma das características mais mencionadas como fontes de degradação ou rejeição na madeira de eucalipto na Austrália (Amaral, 1991). Esse defeito afeta não só a aparência da superfície das peças, mas também suas propriedades mecânicas. As bolsas de resina também prejudicam a madeira para lâminas e painéis.

Conforme Hawley \& Smith (1972), o valor e a utilidade da madeira de povoamentos manejados são reduzidos mais pelos nós e pelas distorções da grã do que por qualquer outro fator. Os ramos, depois de findarem sua atividade fisiológica, raramente caem, pois sua presença não constitui uma desvantagem particular para a sobrevivência da árvore. Assim, a desrama artificial é realizada com o intuito de aumentar a qualidade do produto final, obtendo-se madeira limpa em partes do tronco, que de outra forma só produziriam material de qualidade inferior.
Em um experimento realizado por Schilling et al. (1998), observou-se a influência de diferentes intensidades de desrama sobre a quantidade de nós em Pinus elliottii. Foram retiradas duas toras para obter os corposde-prova, nos quais foram determinados o número e o tamanho dos nós. Foi constatado que na primeira tora a maior quantidade média de nós foi obtida quando se realizou desrama artificial até $50 \%$ da altura total. Para a segunda tora, a maior quantidade de nós foi obtida com o tratamento em que foi realizada a desrama seca, enquanto o tratamento no qual a desrama foi até $40 \%$ da altura total apresentou menor valor.

Couto (1995) afirmou que as desramas devem ser realizadas o mais cedo possível, para obter uma madeira isenta de nós ou de nós de pequenas dimensões. No caso das plantações de eucalipto, a idade em que os ramos estão verdes varia de 1,5 a 3 anos, dependendo do ritmo de crescimento do povoamento. Nessa idade, faz-se a primeira desrama até 2 ou $3 \mathrm{~m}$ de altura, o que corresponderia a cerca de $50 \%$ da copa.

Segundo Fonseca (1979), a desrama apresenta efeitos benéficos sobre a forma das árvores, tornando-as mais cilíndricas. Segundo Kramer \& Kozlowski (1960), tal fato ocorre porque a diminuição do crescimento em diâmetro é maior junto ao solo do que a alturas mais elevadas.

Kozlowski (1971), explicando o efeito da desrama sobre a conicidade, relatou que o crescimento cambial na base do tronco e os acréscimos do xilema após a desrama começam a se concentrar na região não-desgalhada. Assim, a desrama tende a reduzir a conicidade dos troncos, mas seus efeitos dependerão sempre da severidade com que é aplicada e das características das copas das árvores selecionadas.

Na prática, a conicidade é considerada defeito quando a partir do segundo metro, medido até a copa, o diâmetro diminui mais de $1 \mathrm{~cm}$ por metro de comprimento. Este defeito pode ser em parte controlado por medidas silviculturais adequadas, como podas e espaçamento (IBDF, 1984).

Dentre os parâmetros de qualidade sobressai-se também a densidade básica da madeira, que pode ser utilizada como índice seguro para avaliar o tipo de madeira produzida diante de suas correlações com as diferentes propriedades físico-mecânicas, estando, portanto, associada também às mais variadas formas de sua utilização (Brasil et al., 1982). 
Larson (1962) e Fielding (1965) mencionaram o efeito favorável da desrama artificial em acelerar a transição de madeira de lenho inicial para madeira de lenho tardio na região desramada, conseqüentemente nessa região a densidade tem maior valor. Montagna et al. (1990) detectaram tendência de crescimento dos valores da densidade média por efeito dos tratamentos de desrama, especialmente para os pontos situados na base das árvores $(0,30 \mathrm{~m})$. Este fato deve-se fundamentalmente à diminuição do crescimento do tecido primaveril.

Há evidências de que a redução do tecido fotossintético, pela retirada dos ramos vivos da copa, resulta em diminuição da produção de lenho inicial na madeira formada no tronco, influenciando assim os seus valores (Elliott, 1970).

Neste contexto, o objetivo do presente trabalho foi avaliar o efeito de diferentes alturas de desrama artificial sobre a qualidade da madeira, em função da densidade básica, quantidade de nós, bolsas de resina e conicidade de diferentes clones de eucalipto, em sistema agrossilvipastoril.

\section{MATERIAL E MÉTODOS}

\subsection{Descrição da Área Experimental}

Este estudo foi conduzido em uma área experimental de 37,5 ha na fazenda Riacho, pertencente à Companhia Mineira de Metais S.A. (CMM), do Grupo Votorantim, município de Paracatu-MG.

A CMM tem cerca de 13.000 ha de eucalipto plantado em condições de monocultivo, no espaçamento $3 \times 2 \mathrm{~m}$, para produzir madeira para energia, e cerca de 1.000 ha de clones híbridos naturais de Eucalyptus urophylla S.T. Blake, Eucalyptus grandis W.Hill ex Maiden, Eucalyptus saligna Smith e Eucalyptus camaldulensis Dehnh., plantados em consórcio com arroz, soja e pastagem, no espaçamento $10 \mathrm{x} 4 \mathrm{~m}$, objetivando produzir madeira para serraria e para energia (Oliveira et al., 2000).

O sistema agrossilvipastoril implantado inicia-se com o plantio de eucalipto e arroz no ano zero, seguido do plantio de soja no ano 1 e do plantio de capim no ano 2. Assim, o período de engorda de bois começa no terceiro ano e termina no $11^{\circ}$ ano, época em que será feito o corte do eucalipto.

Segundo Antunes (1986), o clima da região é tropical úmido de savana, com inverno seco e verão chuvoso, portanto do tipo Aw na classificação de Köppen. A temperatura média anual é de $22,6^{\circ} \mathrm{C}$, tendo uma média mensal de $18^{\circ} \mathrm{C}$ na estação mais fria e $29,1^{\circ} \mathrm{C}$ na mais quente. A precipitação média anual é de $1.450 \mathrm{~mm}$, apresentando nos meses mais secos precipitações médias mensais inferiores a $60 \mathrm{~mm}$. A vegetação é constituída por cerrados, representada por seus vários tipos, desde campos a cerradões e florestas ciliares subperenifólias, principalmente nas proximidades dos rios, desenvolvida sobre solos derivados de basalto (Golfari, 1975). O solo predominante na área experimental é do tipo Latossolo Vermelho-Amarelo distrófico.

\subsection{Instalação, Tratamentos e Delineamento Experimental}

Para realização deste trabalho foram utilizados três clones de híbridos naturais do gênero Eucalyptus, sendo dois clones de E. urophylla S.T.Blake (código 13 e 44) e um de E. camaldulensis Dehnh. (código 06). Estes clones foram obtidos de árvores superiores dos plantios comerciais da Companhia Mineira de Metais (CMM).

O experimento foi instalado em dezembro de 1994 , no esquema de exploração de sistema agrossilvipastoril. No terceiro ano após o plantio, nas repetições de cada clone, foram aplicados os seguintes tratamentos: $T_{1}$ - sem desrama; $\mathrm{T}_{2}$ - desrama até $2 \mathrm{~m} ; \mathrm{T}_{3}$ - desrama até $4 \mathrm{~m} ; \mathrm{e} \mathrm{T}_{4}$ - desrama até $6 \mathrm{~m}$.

As parcelas experimentais foram alocadas dentro da área, respeitando-se um delineamento experimental de blocos casualizados com três repetições, tendo os tratamentos sido dispostos em esquema de parcelas subdivididas. As subparcelas experimentais foram formadas por oito linhas de nove árvores, totalizando 72 árvores, sendo estas dispostas em espaçamento de 10 m nas entrelinhas e $4 \mathrm{~m}$ entre árvores. As 52 árvores externas foram consideradas como bordadura, sendo a área útil de cada subparcela constituída de $800 \mathrm{~m}^{2}$, ocupada por 20 árvores centrais de eucalipto, dispostas em quatro linhas de cinco plantas cada. As linhas de plantio foram orientadas no sentido leste - oeste.

\subsection{Avaliação da Qualidade da Madeira}

\subsubsection{Densidade Básica da Madeira}

Supondo não existir variação genética dentro da subparcela, foi amostrada apenas uma árvore, no seu centro. Para determinação da densidade básica as árvores

R. Árvore, Viçosa-MG, v.26, n.3, p.285-297, 2002 
foram abatidas, aos 6 anos de idade (2000), e seccionadas de modo a serem retirados três discos de aproximadamente $5 \mathrm{~cm}$ de espessura, ao longo do tronco, ou seja, na base, a $3 \mathrm{~m}$ e a $6 \mathrm{~m}$ de altura. Após a retirada dos discos mensuraram-se os seus diâmetros, e, logo em seguida, estes foram devidamente identificados e acondicionados em sacos plásticos. Foram obtidas também duas toras de $3 \mathrm{~m}$ de comprimento, sendo classificadas como tora 1 e 2 no sentido da base para o topo.

Retirou-se de cada disco uma bagueta radial, passando pela medula, de largura de $2,5 \mathrm{~cm}$, para realização dos ensaios de determinação da densidade básica da madeira.

Para obtenção do perfil de densidade, foram retiradas amostras a cada $1,0 \mathrm{~cm}$ a partir da medula, em direção à casca, para várias alturas do fuste, ou seja, na base, a $3 \mathrm{~m}$ e a $6 \mathrm{~m}$ da altura total da tora. Tais amostras retiradas das baguetas mediram aproximadamente $1,0 \mathrm{x}$ 2,5 x 5,0 cm, sendo a última dimensão no sentido longitudinal da árvore.

Na determinação do volume das amostras, na condição saturada ou verde, utilizou-se o método da balança hidrostática (ASTM, 1984), mas com a substituição do mercúrio pela água, pois os corpos-de-prova se encontravam saturados. Para determinação da massa verde e seca em estufa, utilizou-se uma balança de precisão de 0,01 g. Para secagem completa das amostras utilizou-se uma estufa de ventilação forçada e controle automático, a uma temperatura de $105 \pm 2^{\circ} \mathrm{C}$, até atingirem peso constante.

A densidade básica média de cada árvore foi calculada como sendo a média ponderada da densidade de cada disco, utilizando como fator de ponderação o volume da tora compreendido entre dois discos sucessivos, calculado segundo a fórmula de Smalian (Scolforo \& Figueiredo Filho, 1998).

\subsubsection{Conicidade}

Para determinar a conicidade das árvores, foram obtidos os diâmetros da extremidade inferior e superior das toras. A conicidade foi calculada pela divisão da diferença entre os dois diâmetros pelo comprimento da tora $(6 \mathrm{~m})$.

\subsubsection{Nós e Bolsas de Resina}

As toras oriundas de cada parcela, medindo 3,0 m de comprimento, foram desdobradas na serraria da
Companhia Mineira de Metais - CMM. Retirou-se uma tábua radial, passando pela medula no centro de cada tora, com espessura igual a 5,0 cm, além de outras quatro tábuas tangenciais, sendo duas representando a região intermediária entre a medula e a periferia da tora e as outras duas representando a região periférica.

As tábuas foram identificadas, e em cada uma delas fez-se a contagem dos nós, do diâmetro e da nodosidade. A nodosidade representa a área total que os nós ocupam em relação à área total das peças. De acordo com IBDF (1984), o grau de nodosidade é considerado defeito somente se o seu valor for superior a $3 \%$.

Após as observações dos nós, verificou-se também a ocorrência de bolsas de resina, que foram quantificadas nas tábuas obtidas.

\subsection{Análise Estatística}

Os valores obtidos da densidade básica da madeira, da conicidade e dos nós (número, diâmetro e nodosidade) e o número de bolsas de resina foram submetidos à análise de variância, de acordo com o modelo linear adequado para o experimento em esquema de parcelas subdivididas.

Com a finalidade de avaliar a densidade básica ao longo da tora, considerou-se a posição de retirada do disco como um fator a ser estudado, caracterizado por três posições: base, $3 \mathrm{~m}$ e $6 \mathrm{~m}$.

Com a finalidade de caracterizar as madeiras provenientes de toras da base e do ápice, foram consideradas as duas diferentes posições da tora como um tipo de tratamento.

Quando os efeitos dos tratamentos (clone, altura de desrama, posição no tronco e toras) se mostraram significativos pelo teste de $\mathrm{F}$, foram realizadas as comparações de médias por meio do teste de Scott \& Knott (1974), a 5\% de probabilidade, para cada característica.

\section{RESULTADOS E DISCUSSÃO}

\subsection{Densidade Básica da Madeira}

Como característica tecnológica mais estudada e difundida, a densidade básica é de grande importância na interpretação das demais propriedades da madeira, estando, portanto, associada também às mais variadas 
formas de sua utilização. A variabilidade da densidade básica da madeira por árvore de eucalipto é confirmada pela análise de variância (Quadro 1), tendo sido observado que ambos os fatores apresentaram diferenças significativas, pelo teste de F.

Os valores médios obtidos para densidade básica por árvore para os três clones, nas diferentes alturas de desrama, estão apresentados no Quadro 2.

De acordo com o Quadro 2, o clone que apresentou maior densidade básica por árvore $\left(0,54 \mathrm{~g} / \mathrm{cm}^{3}\right)$ foi o Eucalyptus camaldulensis (clone 06), diferindo-se estatisticamente dos demais clones, que apresentaram valores médios de 0,50 e $0,49 \mathrm{~g} / \mathrm{cm}^{3}$, obtidos pelos clones de E. urophylla 13 e 44, respectivamente. Esse resultado evidencia que podem ser obtidos ganhos com a seleção do melhor material genético. A densidade básica média por árvore dos três clones avaliados foi de $0,51 \mathrm{~g} / \mathrm{cm}^{3}$. Resultados semelhantes foram obtidos por Ribeiro \& Zani Filho (1993), que estudando a variação da densidade básica entre espécies/procedências de eucalipto verificaram que para o $E$. urophylla a densidade básica da madeira foi em média superior a $0,51 \mathrm{~g} / \mathrm{cm}^{3}$. O mesmo foi relatado por Gouvêa et al., (1997), que encontraram valores para densidade básica da madeira para E. urophylla variando de 0,55 a $0,57 \mathrm{~g} / \mathrm{cm}^{3}$, para árvores de 6 anos de idade.

Observa-se também no Quadro 2, para as diferentes alturas de desrama, que apenas o tratamento sem desrama apresentou diferença significativa em relação às demais alturas para densidade básica por árvore, destacando-se

Quadro 1 - Resumo da análise de variância para densidade básica $\left(\mathrm{g} / \mathrm{cm}^{3}\right)$ da madeira por árvore aos 6 anos de idade

Table 1 - Summary of the analysis of variance for wood basic density $\left(\mathrm{g} / \mathrm{cm}^{3}\right)$ of each tree at 6 years of age

\begin{tabular}{|l|c|l|}
\hline \multicolumn{1}{|c|}{ FV } & GL & \multicolumn{1}{c|}{ QM } \\
\hline Bloco & 2 & 0,000136 \\
Clone (A) & 2 & $0,008103 * *$ \\
Erro & 4 & 0,000132 \\
Altura de Desrama (B) & 3 & $0,000478 *$ \\
A x B & 6 & 0,000214 \\
Erro & 18 & 0,000107 \\
\hline CV (\%) & 2,24 & \\
\hline
\end{tabular}

$\mathrm{FV}=$ fontes de variação, $\mathrm{GL}=$ graus de liberdade, $\mathrm{QM}=$ quadrado médio, $\mathrm{CV}=$ coeficiente de variação, $* *=$ significativo a $1 \%$ de probabilidade $\mathrm{e}^{*}=$ significativo a $5 \%$ de probabilidade. com a maior média $\left(0,52 \mathrm{~g} / \mathrm{cm}^{3}\right)$. Para as demais alturas, a média foi de $0,51 \mathrm{~g} / \mathrm{cm}^{3}$, devendo ser ressaltado que estas não diferiram estatisticamente entre si.

Há evidências de que a redução do tecido fotossintético, pela retirada dos ramos vivos da copa, causa redução na produção de lenho inicial na madeira formada no tronco, influenciando assim a densidade básica da madeira de espécies do gênero Pinus (Elliott, 1970). Montagna et al. (1990) verificaram que as diferentes intensidades de desrama artificial não interferiram de forma significativa no desenvolvimento da densidade básica da madeira de Pinus elliottii Engelm. var. elliottii, embora tenha ocorrido tendência de aumento com a severidade da operação.

Do ponto de vista tecnológico, tão importante como o estudo da variação individual é o diagnóstico da variabilidade dentro da árvore, tanto no sentido radial (medulacasca) como no sentido longitudinal (base-topo).

O Quadro 3 mostra a análise de variância realizada para densidade básica da madeira nas diferentes posições ao longo da árvore

Constatou-se (Quadro 3) uma diferença altamente significativa entre os clones e uma significativa, entre as alturas de desrama e as posições ao longo do fuste. Evidencia-se, também, um efeito significativo das interações clone x posição e alturas de desrama x posição. Os valores médios para densidade básica da madeira em cada posição do fuste, e nas diferentes alturas de desrama, estão apresentados no Quadro 4.

Quadro 2 - Valores médios para densidade básica $\left(\mathrm{g} / \mathrm{cm}^{3}\right)$ por árvore, em função dos clones de eucaliptos e das alturas de desrama artificial aos 6 anos de idade

Table 2 - Average values for basic density $\left(\mathrm{g} / \mathrm{cm}^{3}\right)$ per tree, in function of the eucalypt clones and heights of artificial pruning at 6 years of age

\begin{tabular}{|c|c|c|c|c|}
\hline \multirow{2}{*}{$\begin{array}{c}\text { Altura de } \\
\text { Desrama (m) }\end{array}$} & \multicolumn{3}{|c|}{ Clone } & \multirow{2}{*}{ Média } \\
\cline { 2 - 4 } & 13 & 44 & 06 & \\
\hline 0 & $0,52 \mathrm{aB}$ & $0,51 \mathrm{aB}$ & $0,54 \mathrm{aA}$ & $0,52 \mathrm{a}$ \\
2 & $0,50 \mathrm{bB}$ & $0,49 \mathrm{bB}$ & $0,54 \mathrm{aA}$ & $0,51 \mathrm{~b}$ \\
4 & $0,49 \mathrm{bB}$ & $0,49 \mathrm{bB}$ & $0,54 \mathrm{aA}$ & $0,51 \mathrm{~b}$ \\
6 & $0,50 \mathrm{bB}$ & $0,49 \mathrm{bB}$ & $0,54 \mathrm{aA}$ & $0,51 \mathrm{~b}$ \\
\hline Média & $0,50 \mathrm{~B}$ & $0,49 \mathrm{~B}$ & $0,54 \mathrm{~A}$ & \\
\hline
\end{tabular}

Médias seguidas de mesmas letras minúsculas na coluna e maiúsculas na linha não diferem estatisticamente entre si pelo teste Scott e Knott, a 5\% de probabilidade.

R. Árvore, Viçosa-MG, v.26, n.3, p.285-297, 2002 
Quadro 3 - Resumo da análise de variância para densidade básica $\left(\mathrm{g} / \mathrm{cm}^{3}\right)$ da madeira nas diferentes posições ao longo do tronco, aos 6 anos de idade

Table 3 - Summary of the analysis of variance for wood basic density $\left(\mathrm{g} / \mathrm{cm}^{3}\right)$ under the different positions along the log at 6 years of age

\begin{tabular}{|l|c|l|}
\hline \multicolumn{1}{|c|}{ FV } & GL & \multicolumn{1}{|c|}{ QM } \\
\hline Bloco & 2 & 0,000429 \\
Clone (A) & 2 & $0,026723 * *$ \\
Erro (A) & 4 & 0,000683 \\
Altura de Desrama (B) & 3 & $0,001689 *$ \\
Posição (C) & 2 & $0,001715^{*}$ \\
A x B & 6 & 0,001138 \\
A x C & 4 & $0,001536 *$ \\
B x C & 6 & $0,001230^{*}$ \\
A x B x C & 12 & 0,000902 \\
Erro (B) & 66 & 0,000518 \\
\hline CV (\%) & 5,06 & \\
\hline
\end{tabular}

$\mathrm{FV}=$ fontes de variação, $\mathrm{GL}=$ graus de liberdade, $\mathrm{QM}=$ quadrado médio, $\mathrm{CV}=$ coeficiente de variação, $* *=$ significativo a $1 \%$ de probabilidade $\mathrm{e}^{*}=$ significativo a $5 \%$ de probabilidade.

Quadro 4 - Valores médios de densidade básica da madeira de clones de eucaliptos $\left(\mathrm{g} / \mathrm{cm}^{3}\right)$ nas diferentes alturas de desrama artificial ao longo do fuste, aos 6 anos de idade

Table 4 - Wood basic density $\left(\mathrm{g} / \mathrm{cm}^{3}\right)$ average values of eucalypt clones at different heights of artificial pruning, along the stem at 6 years of age

\begin{tabular}{|c|c|c|c|c|}
\hline Altura de & \multicolumn{3}{|c|}{ Posição no Fuste } & \multirow{2}{*}{ Média } \\
\cline { 2 - 4 } Desrama $(\mathrm{m})$ & Base & $3 \mathrm{~m}$ & $6 \mathrm{~m}$ & \\
\hline 0 & $0,53 \mathrm{a}$ & $0,51 \mathrm{a}$ & $0,53 \mathrm{a}$ & $0,52 \mathrm{a}$ \\
2 & $0,51 \mathrm{a}$ & $0,50 \mathrm{a}$ & $0,51 \mathrm{~b}$ & $0,51 \mathrm{~b}$ \\
4 & $0,51 \mathrm{a}$ & $0,50 \mathrm{a}$ & $0,50 \mathrm{~b}$ & $0,51 \mathrm{~b}$ \\
6 & $0,51 \mathrm{a}$ & $0,49 \mathrm{a}$ & $0,54 \mathrm{a}$ & $0,51 \mathrm{~b}$ \\
\hline Média & $0,52 \mathrm{~A}$ & $0,51 \mathrm{~B}$ & $0,52 \mathrm{~A}$ & \\
\hline
\end{tabular}

Médias seguidas de mesmas letras maiúsculas na linha e minúsculas na coluna não diferem estatisticamente entre si pelo teste Scott e Knott, a 5\% de probabilidade.

Observa-se (Quadro 4) que o tratamento sem desrama apresentou valor médio superior para densidade básica, em relação aos demais tratamentos, que não diferiram entre si. As comparações entre as médias demonstram que somente na extremidade $(6 \mathrm{~m})$ do fuste foi observada diferença significativa entre as alturas de desrama, devendo ser ressaltado que nos tratamentos sem desrama e desrama a $6 \mathrm{~m}$ obteve-se uma densidade básica média de $0,53 \mathrm{e}$ $0,54 \mathrm{~g} / \mathrm{cm}^{3}$, respectivamente, superiores aos demais tratamentos. Vale a pena ressaltar que ambos não apresentaram nenhuma diferença significativa entre si, diferindo apenas dos tratamentos de desrama a 2 e $4 \mathrm{~m}$ de altura.
Oliveira (1997) detectou diferenças significativas na densidade básica ao longo da árvore de algumas espécies de Eucalyptus, exceto para o E. citriodora.

Com base nos valores médios obtidos (Quadro 5), pode-se constatar a superioridade do clone 06 de E. camaldulensis, com a densidade básica da madeira apresentando a seguinte ordem decrescente: clone 06 de E. camaldulensis $\left(0,54 \mathrm{~g} / \mathrm{cm}^{3}\right)$, clone 13 de E. urophylla $\left(0,50 \mathrm{~g} / \mathrm{cm}^{3}\right)$ e clone 44 de E. urophylla $\left(0,49 \mathrm{~g} / \mathrm{cm}^{3}\right)$, tendo os dois clones de E. urophylla não diferido entre si.

Observa-se (Quadro 5) que houve diminuição da densidade básica média da base para o meio do tronco ( $3 \mathrm{~m})$ e aumento desta do meio para a extremidade considerada $(6 \mathrm{~m})$.

Uma síntese do estudo detalhado da variação radial (medula-casca) da densidade básica da madeira dos três clones de eucaliptos, nas diferentes alturas de desrama e nas diferentes posições ao longo do tronco, é mostrada nas Figuras 1 e 2.

Os gráficos das Figuras 1 e 2 mostram uma tendência geral para todos os clones, em que suas madeiras possuem densidade básica mais baixa na região da medula. Apesar do comportamento geral, os gráficos mostram que cada espécie possui um perfil de variação de densidade específico, variando para cada posição do tronco e nas diferentes alturas de desrama. Nos três clones avaliados, pode ser observada a tendência de aumento da densidade básica da madeira no sentido radial do tronco. Por outro lado, verifica-se que até aos 6 anos de idade não houve tendência de estabilização dos valores de densidade básica

Quadro 5 - Valores médios para densidade básica $\left(\mathrm{g} / \mathrm{cm}^{3}\right)$ dos três clones de eucaliptos nas diferentes posições do fuste, aos 6 anos de idade

Table 5 - Average values for basic density $\left(\mathrm{g} / \mathrm{cm}^{3}\right)$ of the three eucalypt clones under the different positions of the stem at the 6 years of age

\begin{tabular}{|c|c|c|c|c|}
\hline \multirow{2}{*}{ Clone } & \multicolumn{3}{|c|}{ Posição no Fuste } & \multirow{2}{*}{ Média } \\
\cline { 2 - 4 } & Base & $3 \mathrm{~m}$ & $6 \mathrm{~m}$ & \\
\hline 13 & $0,51 \mathrm{bA}$ & $0,50 \mathrm{bA}$ & $0,50 \mathrm{bA}$ & $0,50 \mathrm{~b}$ \\
44 & $0,51 \mathrm{bA}$ & $0,49 \mathrm{bB}$ & $0,49 \mathrm{bB}$ & $0,49 \mathrm{~b}$ \\
06 & $0,54 \mathrm{aB}$ & $0,54 \mathrm{aB}$ & $0,56 \mathrm{aA}$ & $0,54 \mathrm{a}$ \\
\hline Média & $0,52 \mathrm{~A}$ & $0,51 \mathrm{~B}$ & $0,52 \mathrm{~A}$ & \\
\hline
\end{tabular}

Médias seguidas de mesmas letras minúsculas na coluna e maiúsculas na linha não diferem estatisticamente entre si pelo teste Scott e Knott, a $5 \%$ de probabilidade. 
da madeira, podendo ser obtidos ganhos adicionais em qualidade da madeira quando as árvores são mantidas no campo por períodos maiores. Esse padrão de variação da densidade básica indica que a camada cambial das árvores desses clones, aos 6 anos de idade, encontra-se formando madeira caracterizada totalmente como juvenil.
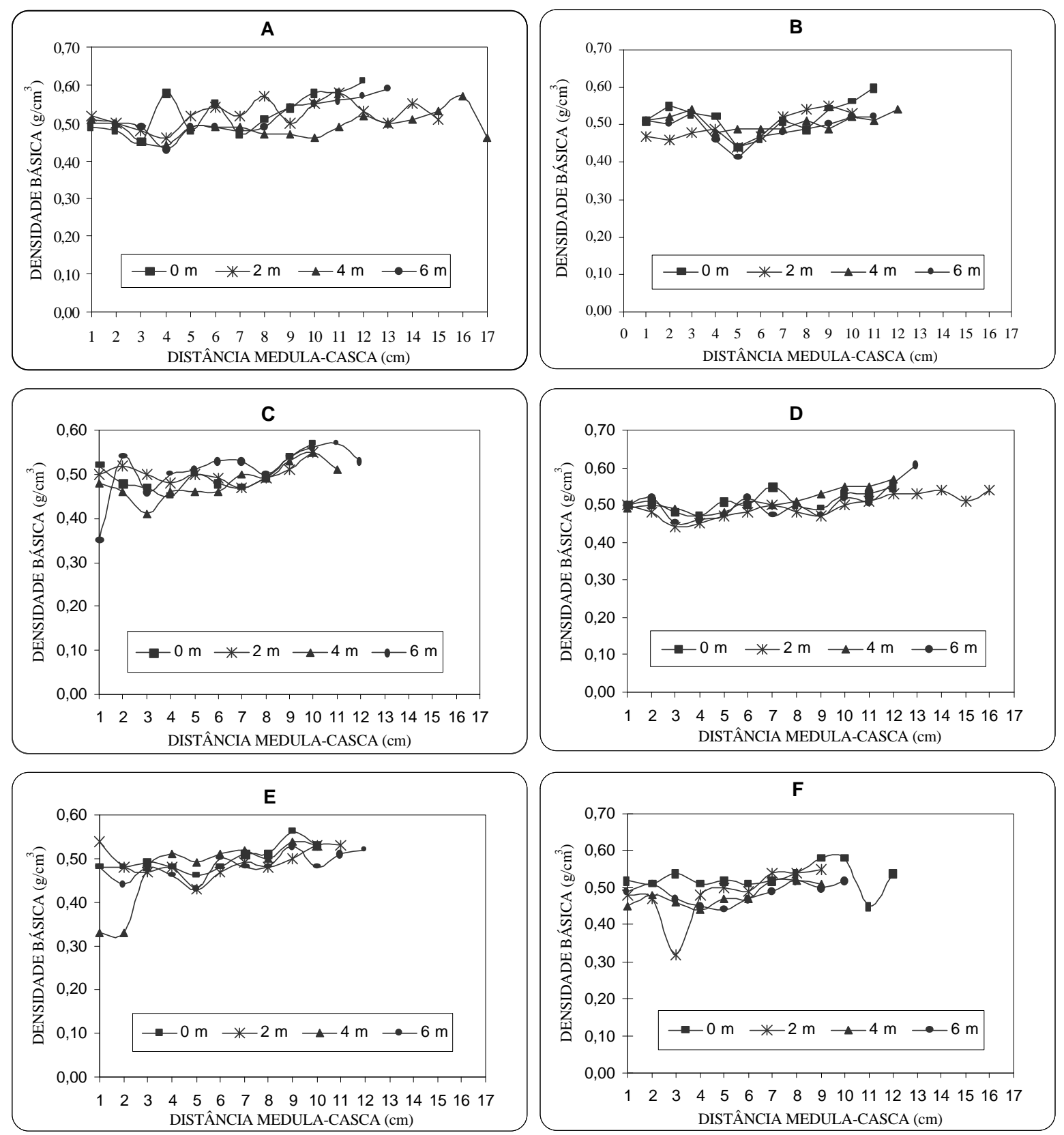

Figura 1 - Representação gráfica da variação da densidade básica média da madeira $\left(\mathrm{g} / \mathrm{cm}^{3}\right)$, para as diferentes alturas de desrama artificial, na direção radial (sentido medula-casca) e nas três posições ao longo do tronco dos clones 13 (A: na base; B: 3 m; C: 6 m) e 44 (D: na base; E: 3 m; F: 6 m) de Eucalyptus urophylla.

Figure 1 - Graphic representation of the wood average basic density variation $\left(\mathrm{g} / \mathrm{cm}^{3}\right)$, for the different heights of artificial pruning, in the radial direction and under the three positions along the stem of the clones 13 (A: on the base; B: $3 \mathrm{~m} ; \mathrm{C}: 6 \mathrm{~m}$ ) and 44 (D: on the base; $E: 3 \mathrm{~m} ; F: 6 \mathrm{~m}$ ) of Eucalyptus urophylla. 

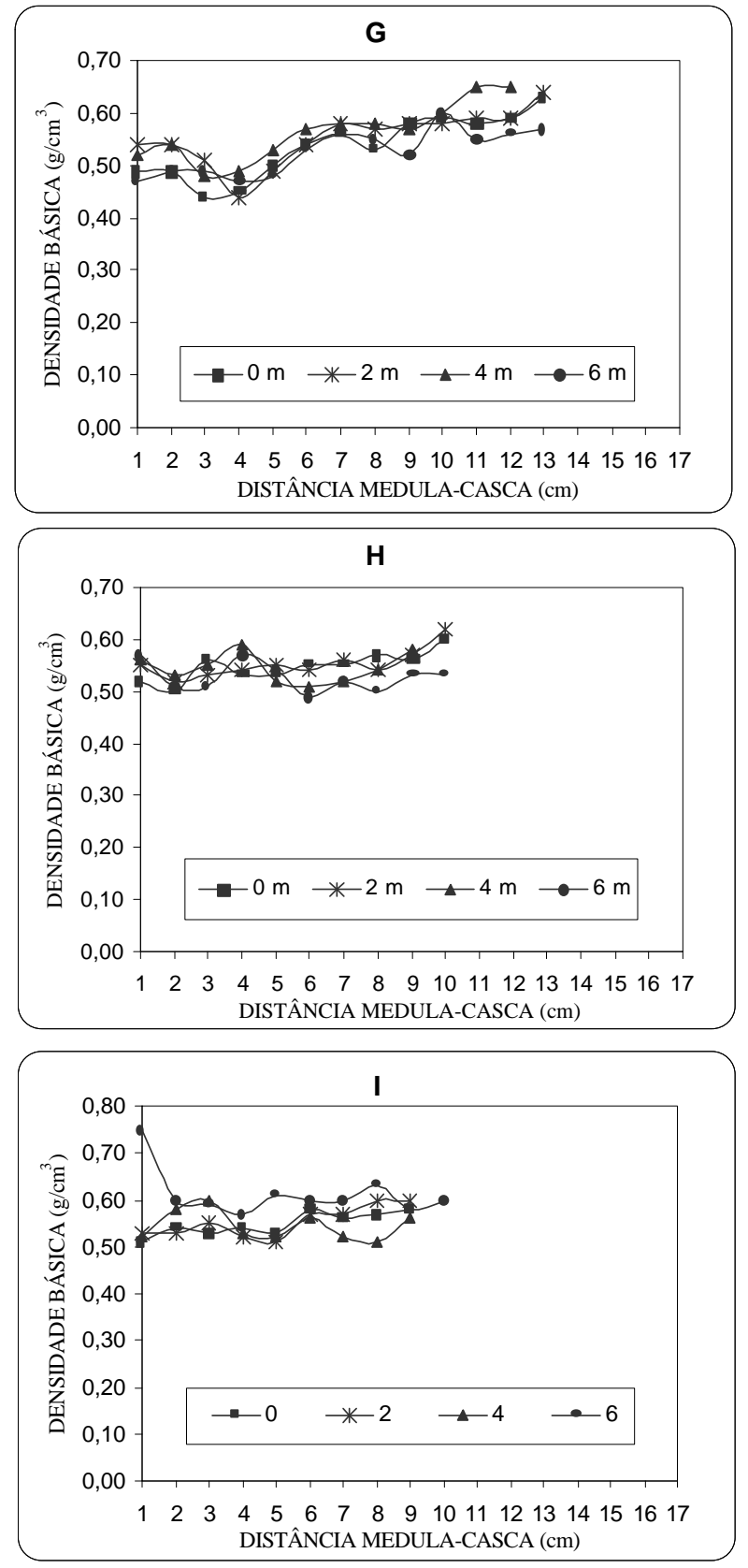

Figura 2 - Representação gráfica da variação da densidade básica média da madeira $\left(\mathrm{g} / \mathrm{cm}^{3}\right)$, para as diferentes alturas de desrama artificial, na direção radial (sentido medula-casca) e nas três posições ao longo do tronco do clone 06 (G: na base; H: 3 m; I: 6 m) de Eucalyptus camaldulensis.

Figure 2 - Graphic representation of the wood average basic density variation $\left(\mathrm{g} / \mathrm{cm}^{3}\right)$, for the different heights of artificial pruning, in the radial direction and under the three positions along the stem of the clone 06 (G: on the base; H: 3 m; I: 6 m) of Eucalyptus camaldulensis.

\subsection{Nós e Bolsas de Resina}

De acordo com a análise de variância (Quadro 6) referente ao número, ao diâmetro dos nós e à nodosidade, pode-se comprovar que os três clones avaliados apresentaram comportamento distinto para todas as características, tendo as toras obtidas desses clones apresentado diferenças significativas em relação ao diâmetro dos nós e à nodosidade. Com relação ao número de nós, não houve diferença significativa entre as toras. As alturas de desrama afetaram de forma significativa o número de nós e a nodosidade. Das interações entre os fatores, constatouse efeito significativo somente da interação clones e toras, para todas as três características avaliadas.

No caso de madeira serrada, o diâmetro dos nós assume importância decisiva. O diâmetro dos nós individuais, principalmente aqueles com grande porção morta absorvida, contribui muito mais para a diminuição das propriedades de resistência de uma peça de madeira do que sua quantidade.

Os resultados obtidos (Quadro 7) indicam que o clone 06 de $E$. camaldulensis diferiu estatisticamente dos demais, apresentando maior quantidade de nós $(43,91)$. No entanto, o diâmetro dos nós e, conseqüentemente, a nodosidade apresentaram valores médios menores, $1,10 \mathrm{~cm}$ e $1,18 \%$, respectivamente, quando comparados aos outros dois clones, 13 e 44 de E. urophylla, que não diferiram estatisticamente entre si em nenhum dos parâmetros relacionados. Este fato é devido à maior ocorrência de desrama natural no clone 06 de E. camaldulensis, pois verificou-se que aos 6 anos de idade já não havia mais galhos inseridos ao tronco até alturas superiores a $6 \mathrm{~m}$, em todas as subparcelas referentes aos diferentes tratamentos aplicados.

Com base nos resultados obtidos no Quadro 8, observou-se que houve diferença significativa entre as toras. Esses resultados demonstram que o diâmetro dos nós e a nodosidade da segunda tora (ápice) apresentam valores médios superiores, $1,78 \mathrm{~cm}$ e $2,15 \%$, respectivamente, aos da primeira tora (base). Essa superioridade é devido ao fato de o diâmetro dos galhos do fuste aumentar, em média, de baixo para cima, até o início da copa, ou seja, o diâmetro médio dos galhos aumenta com a sua altura no tronco.

Os valores médios do número, do diâmetro dos nós e da nodosidade encontrados nas peças estão relacionados no Quadro 9, para a primeira e segunda tora. 
Quadro 6 - Resumo da análise de variância para os valores médios do número de nós, do diâmetro dos nós e da nodosidade (\%), por tora, em função dos clones de eucalipto e das alturas de desrama artificial, aos 6 anos de idade

Table $\boldsymbol{6}$-Summary of the variance analysis for the average values of number of knots, diameter of knots and knottiness (\%), per stem, in function of the eucalypt clones and heights of artificial pruning, at 6 years of age

\begin{tabular}{|l|c|c|c|c|}
\hline \multirow{2}{*}{ FV } & \multirow{2}{*}{ GL } & \multicolumn{3}{|c|}{ QM } \\
\cline { 3 - 5 } & & Número & Diâmetro (cm) & Nodosidade $(\%)$ \\
\hline Bloco & 2 & 133,791 & 0,04823 & 0,17862 \\
Clone (A) & 2 & $1.554,04 * *$ & $2,88751 * *$ & $4,66540 * *$ \\
Erro (A) & 4 & 9,83333 & 0,13041 & 0,32335 \\
Altura de Desrama (B) & 3 & $586,458 * *$ & 0,06033 & $1,87004 *$ \\
Tora (C) & 1 & 36,1250 & $6,09586 * *$ & $15,2517 *$ \\
B x C & 3 & 77,4212 & 0,00854 & 0,02572 \\
A x B & 6 & 47,4305 & 0,10324 & 0,39334 \\
A x C & 2 & $205,041 *$ & $0,29356 *$ & $5,59642 *$ \\
A x B x C & 6 & 18,9490 & 0,02783 & 0,37042 \\
Erro (B) & 42 & 62,4226 & 0,08666 & 0,41234 \\
\hline CV (\%) & & 9,06 & 24,05 & 33,63 \\
\hline
\end{tabular}

$\mathrm{FV}=$ fontes de variação, $\mathrm{GL}=$ graus de liberdade, $\mathrm{QM}=$ quadrado médio, $\mathrm{CV}=$ coeficiente de variação, $* *=$ significativo a $1 \%$ de probabilidade $\mathrm{e}^{*}=$ significativo a $5 \%$ de probabilidade.

Quadro 7 - Valores médios do número de nós, do diâmetro de nós $(\mathrm{cm})$ e da nodosidade $(\%)$ dos três clones avaliados, aos 6 anos de idade

Table 7 -Average values of number of knots, diameter of knots (cm) and knottiness (\%), of the three evaluated clones, at 6 years of age

\begin{tabular}{|c|c|c|c|}
\hline Clone & Número & Diâmetro $(\mathrm{cm})$ & Nodosidade (\%) \\
\hline 13 & $29,95 \mathrm{~b}$ & $1,73 \mathrm{a}$ & $1,97 \mathrm{a}$ \\
44 & $30,00 \mathrm{~b}$ & $1,67 \mathrm{a}$ & $1,91 \mathrm{a}$ \\
06 & $43,91 \mathrm{a}$ & $1,10 \mathrm{~b}$ & $1,18 \mathrm{~b}$ \\
\hline
\end{tabular}

Médias seguidas de mesmas letras minúsculas na coluna não diferem estatisticamente entre si pelo teste Scott e Knott, a 5\% de probabilidade.

Quadro 8 - Valores médios do diâmetro dos nós $(\mathrm{cm})$ e das nodosidade (\%) das toras, aos 6 anos de idade

Table 8 - Average values of diameter of knots (cm) and knottiness (\%) of the stems, at 6 years of age.

\begin{tabular}{|c|c|c|}
\hline Tora & Diâmetro $(\mathrm{cm})$ & Nodosidade $(\%)$ \\
\hline 1 (base) & $1,20 \mathrm{~b}$ & $1,23 \mathrm{~b}$ \\
2 (ápice) & $1,78 \mathrm{a}$ & $2,15 \mathrm{a}$ \\
\hline
\end{tabular}

Médias seguidas de mesmas letras minúsculas na coluna não diferem estatisticamente entre si pelo teste Scott e Knott, a 5\% de probabilidade.
Considerando o diâmetro atingido pelos galhos em determinada idade e altura da árvore, dependendo do material genético utilizado, verificou-se a tendência de o clone 06 de E. camaldulensis concentrar maior quantidade de nós, tanto na primeira quanto na segunda tora, com valores médios de 44,16 e 43,66 nós, respectivamente; os outros dois clones de E. urophylla não apresentaram diferença significativa entre si, em ambas as toras, porém apresentaram valores médios inferiores aos do clone 06. Entretanto, o clone 06 de E. camaldulensis apresentou menores valores médios para o diâmetro dos nós e, conseqüentemente, para a nodosidade nas duas toras avaliadas. Dos dois clones de E. urophylla, o clone 44 destacou-se com menor valor médio de nodosidade na segunda tora $(1,91 \%)$, enquanto na primeira tora apresentou o maior valor médio (1,92\%), superior aos dos clones 06 e 13 (Quadro 9).

Independentemente dos clones avaliados, os tratamentos sem desrama apresentaram valores médios de 43,05 nós e nodosidade de $2,14 \%$, sendo superior aos demais tratamentos de desrama a 2, 4 e 6 m, os quais não diferiram entre si (Quadro 10). Vale ressaltar que nas subparcelas dos tratamentos sem desrama foram encontrados galhos que permaneceram aderidos ao tronco, com diâmetro de até $10 \mathrm{~cm}$, o que justifica sua maior nodosidade.

R. Árvore, Viçosa-MG, v.26, n. 3, p.285-297, 2002 
Quadro 9 - Valores médios do número de nós, do diâmetro dos nós (cm) e da nodosidade (\%), por tora, nos três clones de eucalipto, aos 6 anos de idade

Table 9 -Average values of number of knots, diameter of knots (cm) and knottiness (\%), per stem, in the three eucalypt clones, at 6 years of age.

\begin{tabular}{|c|c|c|c|c|c|c|}
\hline \multirow{2}{*}{ Clone } & \multicolumn{2}{|c|}{ Número } & \multicolumn{2}{|c|}{ Diâmetro $(\mathrm{cm})$} & \multicolumn{2}{|c|}{ Nodosidade (\%) } \\
\hline & Tora 1 & Tora 2 & Tora 1 & Tora 2 & Tora 1 & Tora 2 \\
\hline 13 & $28,00 \mathrm{~b}$ & $31,91 \mathrm{~b}$ & $1,30 \mathrm{a}$ & $2,14 \mathrm{a}$ & $1,01 \mathrm{~b}$ & $2,93 \mathrm{a}$ \\
\hline 44 & $33,83 \mathrm{~b}$ & $26,91 \mathrm{~b}$ & $1,45 \mathrm{a}$ & $1,89 \mathrm{~b}$ & $1,92 \mathrm{a}$ & $1,91 \mathrm{~b}$ \\
\hline 06 & $44,16 \mathrm{a}$ & $43,66 \mathrm{a}$ & $0,86 \mathrm{~b}$ & $1,33 \mathrm{c}$ & $0,76 \mathrm{~b}$ & $1,60 \mathrm{~b}$ \\
\hline
\end{tabular}

Médias seguidas de mesmas letras minúsculas na coluna não diferem estatisticamente entre si pelo teste Scott e Knott, a 5\% de probabilidade.

Apesar de o tratamento sem desrama apresentar valor médio de nodosidade superior aos dos demais tratamentos, estes não podem ser considerados defeito, pois apresentam nodosidade inferior a $3 \%$, conforme IBDF (1984).

Visando obter maior valor agregado ao produto madeira, com a obtenção de madeiras isentas de nós, deve ser feita a seleção do clone que apresentou menores valores médios para nodosidade, associada à adoção da prática de desrama artificial. Dentro deste contexto, destaca-se o clone 06 de E. camaldulensis, no qual deve ser realizada a desrama em níveis mais inferiores, tendo em vista a redução dos custos na realização de tal prática.

De acordo com a análise de variância para o número de bolsas de resina, constatou-se que esta característica foi altamente influenciada pelo material genético utilizado e pelas posições no tronco, relativas às duas toras avaliadas (Quadro 11).

Quadro 10 - Valores médios do número de nós e nodosidade (\%) nas diferentes alturas de desrama (m), aos 6 anos de idade

Table 10 - Average values of number of knots and knottiness $(\%)$ in the different pruning heights $(m)$, at 6 years of age

\begin{tabular}{|c|c|c|}
\hline $\begin{array}{c}\text { Altura de desrama } \\
(\mathrm{m})\end{array}$ & Número & Nodosidade (\%) \\
\hline 0 & $43,05 \mathrm{a}$ & $2,14 \mathrm{a}$ \\
2 & $31,05 \mathrm{~b}$ & $1,39 \mathrm{~b}$ \\
4 & $31,16 \mathrm{~b}$ & $1,55 \mathrm{~b}$ \\
6 & $33,22 \mathrm{~b}$ & $1,65 \mathrm{~b}$ \\
\hline
\end{tabular}

Médias seguidas de mesmas letras minúsculas na coluna não diferem estatisticamente entre si pelo teste Scott e Knott, a 5\% de probabilidade.
Os resultados obtidos (Quadro 12) comprovam que para o parâmetro em questão o clone 13 de E. urophylla apresentou maior número médio de bolsas de resina $(3,12)$ e os clones 44 de E. urophylla e o clone 06 de E. camaldulensis apresentaram menores valores médios (0,75 e 1,16, respectivamente), ressaltando que ambos não diferiram estatisticamente entre si.

Com relação ao número de bolsas na primeira e segunda tora (Quadro 13), verificou-se a mesma tendência do número de nós, sendo maiores na segunda tora $(2,08)$.

Quadro 11 - Resumo da análise de variância para os valores médios do número de bolsas de resina por tora, em função dos clones de eucalipto e das alturas de desrama artificial, aos 6 anos de idade

Table 11 - Summary of the variance analysis for the average values of number of pitch pockets for each log, in function of the eucalypt clones and heights of artificial pruning, at 6 years of age

\begin{tabular}{|l|c|c|}
\hline \multicolumn{1}{|c|}{ FV } & GL & QM \\
\hline Bloco & 2 & 5,84722 \\
Clone (A) & 2 & $38,5972 * *$ \\
Erro (A) & 4 & 1,59722 \\
Altura de Desrama (B) & 3 & 4,90277 \\
Tora (C) & 1 & $11,6805 *$ \\
B x C & 3 & 3,16203 \\
A x B & 6 & 1,26388 \\
A x C & 2 & 1,93055 \\
A x B x C & 6 & 2,18981 \\
Erro (B) & 42 & 1,85515 \\
\hline CV (\%) & & 75,2 \\
\hline
\end{tabular}

$\mathrm{FV}=$ fontes de variação, $\mathrm{GL}=$ graus de liberdade, $\mathrm{QM}=$ quadrado médio, $\mathrm{CV}=$ coeficiente de variação, $* *=$ significativo a $1 \%$ de probabilidade, $*$ significativo a $5 \%$ de probabilidade. 
Quadro 12 - Valores médios do número de bolsas de resina dos três clones de eucaliptos, aos 6 anos de idade

Table 12 - Average values of number of pitch pockets of the three eucalypt clones, at 6 years of age

\begin{tabular}{|c|c|}
\hline Clone & Número \\
\hline 13 & $3,12 \mathrm{a}$ \\
44 & $0,75 \mathrm{~b}$ \\
06 & $1,16 \mathrm{~b}$ \\
\hline
\end{tabular}

Médias seguidas de mesmas letras minúsculas na coluna não diferem estatisticamente entre si pelo teste Scott e Knott, a 5\% de probabilidade.

Quadro 13 - Valores médios do número de bolsas de resina das duas toras, aos 6 anos de idade

Table 13 - Average values of number of pitch pockets of the two logs, at 6 years of age

\begin{tabular}{|c|c|}
\hline Tora & Número \\
\hline 1 (base) & $1,27 \mathrm{~b}$ \\
2 (ápice) & $2,08 \mathrm{a}$ \\
\hline
\end{tabular}

Médias seguidas de mesmas letras minúsculas na coluna não diferem estatisticamente entre si pelo teste Scott e Knott, a 5\% de probabilidade.

\subsection{Conicidade}

A conicidade é um fator extremamente importante no processamento em serraria ou laminação. Além da diminuição do rendimento, há ainda a diminuição da qualidade e também da produtividade.

A análise de variância, apresentada no Quadro 14, demonstrou que para este índice só ocorreu diferença significativa entre os clones avaliados aos 6 anos de idade (2000). Portanto, as diferentes alturas de desrama realizadas não tiveram nenhum efeito significativo sobre a conicidade das árvores. Esse resultado foi semelhante ao obtido por Campos et al. (1984), que ao analisarem parâmetro semelhante em pesquisa com Pinus taeda e ao avaliarem a variação do fator de forma do tronco com a intensidade de desrama e posição sociológica das árvores desramadas verificaram que não houve diferença significativa de forma entre as árvores submetidas aos diferentes níveis de desrama e nem entre as árvores situadas em diferentes posições sociológicas.

O resultado foi diferente daquele relatado por Kozlowski (1971), que ao explicar o efeito da desrama artificial sobre a conicidade destacou que o crescimento cambial na base do tronco e os acréscimos do xilema após a desrama começam a se concentrar na região não desgalhada. Assim, a desrama tende a diminuir a conicidade dos troncos, mas seus efeitos dependerão sempre da severidade com que é aplicada e das características das copas das árvores. Da mesma forma, Montagna et al. (1996), avaliando os efeitos de diferentes níveis de desrama na conicidade das árvores, verificaram que a desrama interferiu na conicidade somente na base das árvores. De modo geral, conforme mencionado por Caixeta (2000), os troncos são classificados como cônicos quando a diminuição de seu diâmetro, da base em direção à copa, é de mais de $1 \mathrm{~cm}$ por metro linear.

Os valores médios da conicidade para os clones estão apresentados no Quadro 15.

Quadro 14 - Resumo da análise de variância para a conicidade por árvore dentro de cada clone nas diferentes alturas de desrama, aos 6 anos de idade

Table 14 -Summary of the variance analysis for the conicity per tree inside each clone under the different pruning heights, at 6 years of age

\begin{tabular}{|l|c|l|}
\hline \multicolumn{1}{|c|}{ FV } & GL & QM \\
\hline Bloco & 2 & 0,000003 \\
Clone (A) & 2 & $0,000063 * *$ \\
Erro & 4 & 0,000002 \\
Altura de Desrama (B) & 3 & 0,000003 \\
A x B & 6 & 0,000002 \\
Erro & 18 & 0,000002 \\
\hline CV (\%) & 9,85 & \\
\hline
\end{tabular}

$\mathrm{FV}=$ fontes de variação, $\mathrm{GL}=$ graus de liberdade, $\mathrm{QM}=$ quadrado médio, $\mathrm{CV}=$ coeficiente de variação, $* *=$ significativo a $1 \%$ de probabilidade $\mathrm{e}^{*}=$ significativo a $5 \%$ de probabilidade.

Quadro 15 - Valores médios para conicidade $(\mathrm{cm} / \mathrm{m}$ linear) por árvore, em função dos clones de eucalipto e das alturas de desrama artificial, aos 6 anos de idade

Table 15 - Average values for conicity (linear $\mathrm{cm} / \mathrm{m}$ ) per tree, in function of the eucalypt clones and heights of artificial pruning at 6 years of age

\begin{tabular}{|c|c|}
\hline Clone & Conicidade $(\mathrm{cm} / \mathrm{m}$ linear $)$ \\
\hline 13 & $0,01 \mathrm{~b}$ \\
44 & $0,01 \mathrm{~b}$ \\
06 & $0,02 \mathrm{a}$ \\
\hline Média & 0,01 \\
\hline
\end{tabular}

Médias seguidas de mesmas letras minúsculas na coluna não diferem estatisticamente entre si pelo teste Scott e Knott, a 5\% de probabilidade.

R. Árvore, Viçosa-MG, v. 26, n.3, p.285-297, 2002 
De acordo com o Quadro 15, os resultados médios obtidos para este parâmetro de qualidade indicam que a maior conicidade foi apresentada pelo clone 06 de E. camaldulensis $(0,02 \mathrm{~cm} / \mathrm{m})$, diferindo-se estatisticamente dos demais clones de E. urophylla. A média geral obtida foi de $0,01 \mathrm{~cm} / \mathrm{m}$ para conicidade, podendo esta ser considerada baixa, o que garante uma madeira de classe superior e proporciona bom rendimento da madeira para serraria e laminação, pois acarreta a retirada de menor volume de costaneira.

\section{CONCLUSÕES}

- A densidade básica da madeira por árvore apresentou variações significativas entre os clones de eucalipto, permitindo a seleção do melhor material genético, tendo o clone 06 de E. camaldulensis apresentado a maior densidade básica em relação aos clones 13 e 44 de E. urophylla.

- A densidade básica da madeira por árvore apresentou variações significativas para as alturas de desrama artificial.

- A densidade básica ao longo do tronco, sentido longitudinal, apresentou comportamento diferenciado perante os clones e as alturas de desrama.

- O número de nós e a nodosidade foram afetados pelas diferentes alturas de desrama realizadas, enquanto o diâmetro dos nós não sofreu nenhuma variação significativa, tendo sido observada menor nodosidade para o clone 06 de E. camaldulensis.

- $\quad$ C clone 44 de $E$. urophylla apresentou menor número $(0,75)$ de bolsas de resina, devendo ser ressaltado que, de modo geral, o maior número de bolsas de resina encontra-se na tora 2.

- $\quad$ O número de bolsas de resina não foi afetado pelas diferentes alturas de desrama artificial nos clones 13 e 44 de E. urophylla e 06 de E. camaldulensis.

- A conicidade das árvores não apresentou variações significativas entre as alturas de desrama, e sim entre os clones, tendo o clone 06 de E. camaldulensis apresentado maior conicidade $(0,02 \mathrm{~cm} / \mathrm{m}$ linear $)$.

\section{AGRADECIMENTO}

Somos gratos à Companhia Mineira de Metais CMM (Unidade Agroflorestal), pela concessão da área de estudo e todo apoio necessário para realizar este trabalho; à FAPEMIG/FIEMG-IEL, por financiarem o projeto; ao Camilo Lélis Ferreira da Silva e aos engenheiros florestais Antonio Bartolomeu do Vale, José Tarcísio da Silva Oliveira, Dalmo Arantes Barros e Josébio E. Gomes, pelas sugestões e pelo apoio nos trabalhos de campo.

\section{REFERÊNCIA BIBLIOGRÁFICA}

AMARAL, A. C. B. Defeitos na madeira de eucaliptos: Suas causas e possibilidades de redução (revisão bibliográfica). Piracicaba: Escola Superior de Agricultura "Luiz de Queiroz"/LCF, 1991. 25 p.

\section{AMERICAN SOCIETY FOR TESTING AND}

MATERIALS - ASTM. Annual book of A.S.T.M., Standards, Section 4, v. 04.09. Philadelphia: 1984. 734 p.

ANTUNES, F. Z. Caracterização climática do Estado de Minas Gerais: climatologia agrícola. Informe

Agropecuário, v. 12, n. 138, p. 9-13, 1986.

BRASIL, M. A. M. et al. Densidade básica da madeira de Pinus elliottii var. elliottii em três regiões do Estado de São Paulo. Boletim Técnico Instituto Florestal, v. 1, n. 36, p. 9-17, 1982.

BURGER. L. M.; RICHTER, H. G. Anatomia da madeira. São Paulo: Nobel, 1991. 154 p.

CAIXETA, R. P. Qualidade da madeira, classificação e seleção de genótipos de eucalipto. Lavras: Universidade Federal de Lavras, 2000. 94 p. Dissertação (Mestrado em Engenharia Florestal) - Universidade Federal de Lavras, 2000.

CAMPOS, W. O.; SPELTZ, G. E.; CORDEIRO, J. A. O efeito de três níveis de desrama sobre o crescimento volumétrico e forma do fuste em Pinus taeda Linn. In: CONGRESSO FLORESTAL ESTADUAL, 5, 1984, Nova Prata. Anais... Nova Prata: 1984. p.1-12.

COUTO, H. T. Z. Manejo de florestas e sua utilização em serraria. In: SEMINÁRIO INTERNACIONAL DE UTILIZAÇÃO DA MADEIRA DE EUCALIPTO PARA SERRARIA, 1, 1995, São Paulo. Anais... São Paulo: IPT, 1995. p. 21-30.

DEL MENEZZI, C. H. S.; NAHUZ, M. A. R. Comportamento de Eucalyptus grandis W. Hill ex Maiden no Desdobro. Revista Árvore, v. 22, n. 4, p. 563-571, 1998.

ELliOTT, G. K. Wood density in conifers. Technical Communication CAB, v. 8, p. 1-44, 1970. 
FIELDING, J. M. Prunning Pinus taeda in Australia with particular reference to the wood produced. In: MEETING OF SECTION 41; FOREST PRODUCTS. WORKING GROUP OF WOOD AND TREE CHEMISTRY, 1965. Melbourne. Proceedings... Melbourne. IUFRO. 1965. v. 2. p. 1-8.

FONSECA, S. M. Implicações técnica e econômicas na utilização da desrama artificial. Circular Técnica IPEF, n. 46. p. 1-22, 1979.

GOLFARI, L . Zoneamento ecológico do Estado de Minas Gerais para o reflorestamento. Belo Horizonte: PRODEPEF/PNUD/FAO/IBDF/BRA-45, 1975. 65 p. (Série técnica, 3).

GOUVÊA, C. F. et al. Seleção fenotípica por padrão de proporção de casca rugosa persistente em árvores de Eucalyptus urophylla S.T. Blake, visando formação de população base de melhoramento genético: Qualidade da madeira. In: CONFERÊNCIA IUFRO SOBRE SILVICULTURA E MELHORAMENTO DE EUCALIPTO, 1997, Salvador. Anais... Salvador: 1997. p. 355-360.

HAWLEY, R. C.; SMITH, D. M. Silvicultura prática. Barcelona: Omega, 1972. 544 p.

\section{INSTITUTO BRASILEIRO DE DESENVOLVIMENTO}

FLORESTAL - IBDF. Normas para medição e classificação de toras de madeiras de folhosas. Brasília: 1984. 42 p.

KOZLOWSKI, T. T. Growth and development of trees. New York: Academic Press, 1971. 541 p.

KRAMER, P. J.; KOZLOWSKI, T. T. Physiology of trees. New York: MacGraw-Hill, 1960. 642 p.

LARSON, P. R. A biological approach to wood quality. Tappi, v. 6, n. 45, p. 443-448, 1962.
MONTAGNA, R. G. et al. Influência da desrama artificial sobre o crescimento e a densidade básica da madeira de Pinus elliottii var. elliottii. Revista do Instituto Florestal, v. 2, n. 2. p. 157-169, 1990.

MONTAGNA, R.G. et al. Influência da desrama artificial sobre o crescimento e a densidade básica da madeira de Pinus elliottii var. elliottii. Curso em treinamento sobre poda em espécies arbóreas florestais e de arborização urbana. 1. Piracicaba: IPEF/USP, 1996. 90 p.

OLIVEIRA, A. D.; SCOLFORO, J. R. S.; SILVEIRA, V. P. Análise de um sistema agro-silvo-pastoril com eucalipto implantado em região de cerrado. Revista Ciência Florestal, v. 10, n. 1, p. 1-19, 2000.

OLIVEIRA, J. T. S. Caracterização da madeira de eucalipto para a construção civil. São Paulo: Universidade de São Paulo, 1997. 2 v. Tese (Doutorado em Engenharia de Construção Civil e Urbana) - Universidade de São Paulo, 1997.

RIBEIRO, F. A.; ZANI FILHO, J. Variação da densidade básica da madeira em espécies/procedências de Eucalyptus spp. Revista IPEF, n. 46, p. 76-85, 1993.

SCHILLING, A. C. et al. Influência de diferentes intensidades de desrama sobre a porcentagem de lenho tardio e quantidade de nós da madeira de primeiro desbaste de Pinus elliottii Engelman. Santa Maria. Revista Ciência Florestal, v. 8, n. 1, 1998. p. 115-127.

SCOLFORO, J. R. S.; FIGUEIREDO FILHO, A. Biometria florestal: medição e volumetria de árvores florestais. Lavras: UFLA/FAEPE, 1998. 310 p.

SCOTT, A. J.; KNOTT, M. A cluster analysis method for grouping means in the analysis of variance. Biometrics, v. 30, p. 507-512, 1974. 